\title{
Research Paper: Predictive Value of Somatosensation for Manual Dexterity and Upper Limb Motor Function in Stroke Survivors
}

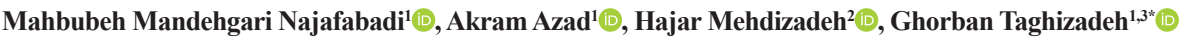 \\ 1. Department of Occupational Therapy, School of Rehabilitation Sciences, Iran University of Medical Sciences, Tehran, Iran. \\ 2. Department of Occupational Therapy, School of Rehabilitation, Tehran University of Medical Sciences, Tehran, Iran. \\ 3. Rehabilitation Research Center, School of Rehabilitation Sciences, Iran University of Medical Sciences, Tehran, Iran.
}

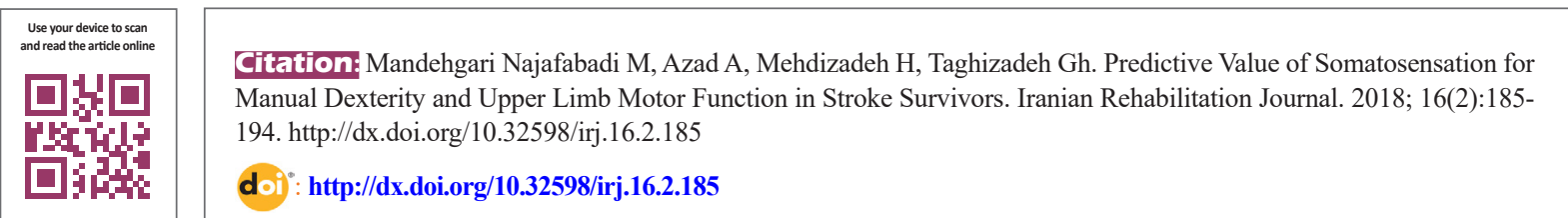

Article info:

Received: 01 Oct 2017

Accepted: 20 Feb 2018

\section{Keywords:}

Correlation, Motor activity, Somatosensory disorders, Stroke, Upper extremity

\begin{abstract}
Objectives: One of the most disabling impairments following stroke is upper limb impairment. Despite the important role of somatosensory function in motor control and high prevalence of somatosensory deficits in stroke survivors, little attention has been paid to its effect on UE motor function in chronic stroke survivors. Thus, the aim of this study is to explore the correlation between different somatosensations and manual dexterity as well as UE motor function in these patients.
\end{abstract}

Methods: In this correlational study, 225 chronic stroke survivors (112 female and 113 male) participated, selected by simple non-probability method among the stroke survivors admitted to the rehabilitation centers in Tehran. The lower-order somatosensations, including light touch and wrist Proprioception, were evaluated by Weinstein Enhanced Sensory Test (WEST) and Wrist Position Sense Test (WPST), respectively. The higher-order somatosensations were measured by static and moving 2-Point Discrimination (s2PD and m2PD, respectively), Hand Active Sensation Test (HAST) and Haptic Object Recognition Test (HORT). Gross and fine manual dexterity and UE motor function were assessed using Box-Block Test (BBT), Purdue Pegboard Test (PPT) and Wolf Motor Function Test (WMFT), respectively.

Results: The multiple regression models showed that WPST alone accounted for the $38.8 \%$ $56.6 \%$ of the variance in manual dexterity and UE motor function. The WEST was the second most significant predictor in all regression models. The HORT, m2PD and HAST explained a small percent of the variance in regression models.

Discussion: These results suggest that treatments that target somatosensory impairments, especially wrist Proprioception and light touch, may be particularly important for improving manual dexterity and UE motor function in chronic stroke survivors.

\section{* Corresponding Author:}

Ghorban Taghizadeh, PhD

Address: Department of Occupational Therapy, School of Rehabilitation Sciences, Iran University of Medical Sciences, Tehran, Iran.

Tel: +98 (910) 1462167

E-mail:taghizadeh.gh@iums.ac.ir;gh_taghizade@yahoo.com 


\section{Introduction}

A

pproximately $70 \%$ of stroke survivors have moderate to severe impairments of Upper Extremity (UE) function and most of them remain with a non-functional UE despite receiving rehabilitation [1]. It has been reported that UE functional impairments are significant contributors to dependency in activities of daily living and participation restrictions [2]. Moreover, somatosensory deficits are also prevalent following stroke, affecting up to $88 \%$ of stroke survivors. Although many studies have been conducted on UE function of stroke survivors, little information exists about the effect of somatosensory deficits on UE function of these patients [3].

Somatosensory information are received by the receptors located in the skin, muscles, ligaments and joints [4]. Then, these information are processed by different brain regions including cerebellum, thalamus, posterior parietal cortex, insula and primary and secondary somatosensory cortices [5]. Somatosensations are classified into the lower and higher order categories. Exteroceptive somatosensation (including pain and light touch) and proprioceptive somatosensation (i.e. position and movement sense) are classified as lower-order somatosensation while somatosensory discrimination (i.e. tactile, weight and texture discrimination, and stereognosis) is considered as higher-order somatosensation [6]. Loss of touch and proprioceptive sensation are prevalent in up to $65 \%$ of stroke survivors [7, 8]. More often, impairments of discrimination and interpretation of sensory stimulus such as stereognosis, weight and texture discrimination are observed in these patients. These somatosensory deficits affect the ability to manipulate objects and identify their different properties [9] and impair sequencing of multi-joint movements $[10,11]$, correcting ongoing movements [12] and controlling goal-directed movements [10] which lead to impaired spontaneous hand use and motor learning $[9,12]$.

Therefore, somatosensory impairments may affect manual dexterity and UE function of stroke survivors, however little investigation has been conducted in this field. Although significant correlation between different types of Somatosensation (including light touch, Proprioception and tactile discrimination) and UE motor function/impairment has been shown in previous studies [13-17], most of these studies have been conducted on the acute and sub-acute stroke survivors and there is little evidence about this correlation in chronic stroke survivors. Only recently, Scalha et al. (2011) investigated this correlation in subjects with chronic stroke and found that tactile sensation of the affected UE was significantly correlated with motor subscale of Fugl-Meyer Assessment (FMA) [16]. However, Scalha et al. pointed out a small sample size $(n=20)$ as a major limitation of their study. Moreover, they did not investigate the predictive value of different types of Somatosensation for UE function. Therefore, this study aimed at comprehensively investigate the correlation between somatosensation (in both lower- and higher-order levels) and manual dexterity as well as UE motor function in chronic stroke survivors. The results of this study would enhance the knowledge of contributing factors in impairments of manual dexterity and UE motor function in chronic stroke survivors which would inform the development of best clinical interventions to improve these impairments.

\section{Methods}

\section{Participants}

In this correlational study, a total of 225 chronic stroke survivors (112 female and 113 male) were included. They were selected by simple non-probability method among the stroke survivors admitted to the rehabilitation centers in Tehran. The study was conducted during the first 8 months of 2016. The inclusion criteria were chronic (>6 months) first ever stroke and the appropriate level of cognitive function (i.e. Mini Mental State Examination score $\geq 21[18,19])$. Participants with other neurological disorders such as Parkinson's disease, orthopedic or rheumatologic disorders and those with visuospatial neglect (i.e. score $\geq 44$ on star cancellation test [20]) were excluded from the study. All participants signed a written consent form, and the protocol was approved by the Ethics Committee of Iran University of Medical Sciences.

\section{Predictors and outcomes}

This study comprehensively explored the extent to which lower and higher-order Somatosensations along with demographic/clinical characteristics could predict gross and fine manual dexterity, and UE motor function in chronic stroke survivors. A demographic questionnaire was used to record the demographic and clinical characteristics including sex, stroke type, affected side, dominant hand, age and time since stroke. Both lowerorder Somatosensations (i.e. light touch and wrist Proprioception) and higher-order Somatosensations (i.e. tactile, weight and texture discrimination as well as haptic performance) were evaluated. The outcomes considered in this study were gross and fine manual dexterity and UE motor function, which were evaluated by Box- 
Block test (BBT), Purdue Pegboard test (PPT) and Wolf motor function test (WMFT), respectively.

\section{Weinstein Enhanced Sensory Test (WEST)}

The light touch threshold was measured by Weinstein Enhanced Sensory Test (WEST) in which the finger tip of the thumb, index and middle fingers of the affected hand were probed by five filaments following the procedure described previously. Each of the three fingers was scored on a $0-5$ scale and the average of the three finger's score was calculated to form the light touch threshold score. The higher WEST score indicates better light touch sensation $[21,22]$. High reliability of the WEST $(\mathrm{r}=0.86, \kappa=0.71-0.79)$ has been reported for assessing light touch threshold in the affected hand of chronic stroke survivors [23].

\section{Wrist Position Sense Test (WPST)}

The Wrist Position Sense Test (WPST) was used to measure wrist Proprioception following the procedure suggested by Carey et al. [24]. Briefly, the assessor moved the participant's wrist to specified position (10 positions for wrist extension and 10 positions for wrist flexion). The participants were required to show perceived angle of her/his wrist following each movement. The average error of 20 positions was considered as a WPST score for each participant. Carey et al. (1996) found that WPST is a reliable measure for evaluating wrist Proprioception in stroke survivors [24].

\section{Two-Point Discrimination test (2PD)}

Tactile discrimination of the thumb, middle and index fingers of the affected hand was evaluated by both static and moving 2-point discrimination test (s2PD and $\mathrm{m} 2 \mathrm{PD}$, respectively). The test was performed using Disk-Criminator and the minimum distance at which the participant could discriminate the 2 point was measured and then it was scored on a $0-3$ scale as follows: $0=16$ $\mathrm{mm}, 1=15-11 \mathrm{~mm}, 2=6-10 \mathrm{~mm}$; and $3=5 \mathrm{~mm}$. In $\mathrm{m} 2 \mathrm{PD}$, the disk criminate was moved from proximal to distal along the long axis of the distal phalanx [21]. The mean score of the three fingers was calculated and its higher value indicates better tactile discrimination. High reliability of the 2PD has been reported previously in stroke survivors [25].

\section{Hand Active Sensation Test (HAST)}

The ability to discriminate weight and texture was assessed by Hand Active Sensation Test (HAST) follow- ing the procedures defined by Williams et al. [26]. The HAST included a familiar objects with similar size and shape and different texture and weight. Participants were asked to manually explore the objects by the affected hand and match them using a matched to sample-forced choice. This matching process was performed twice, once according to the object's texture ( 9 trials) and once according to their weight ( 9 trials). The HAST score was the total number of correct matches (0-18) and higher score indicates better ability to discriminate texture and weight. Williams et al. (2006) showed that HAST is a reliable and valid measure for assessing stroke survivors even for those with no reported sensory deficit [26].

\section{Haptic Object Recognition Test (HORT)}

Haptic performance was evaluated by Haptic Object Recognition Test (HORT). The HORT consisted of five different groups of non-familiar cubic objects $(1.5 \times 2.7 \times 4.7)$ made of LEGO bricks. In each group, objects had a specific number of rectangular bricks in different positions of the sides. In order to facilitate visual discrimination, the constructional differences were highlighted by color. First, in the familiarization phase, one sample object from each group was introduce to the participant and he/she was allowed to familiarize with them by haptic and visual exploration. Then the participant was required to explore a total of 17 objects, hidden in a fabric sac, only through haptic exploration using the affected hand and specify the group of each explored object by placing it in a box behind the specific sample on the table. The participants were asked to perform the test as accurate and as fast as possible. The test was performed after three trials and the average was calculated to determine the HORT score [27].

\section{Box-Block Test (BBT)}

Gross manual dexterity was evaluated using BoxBlock Test (BBT) in which the participants were asked to quickly transfer wooden blocks from one compartment to another using the affected hand. The BBT score was determined by the number of transferred block during 60s. High test-retest reliability and good validity has been reported for the BBT [28].

\section{Purdue Pegboard Test (PPT)}

The Purdue Pegboard Test (PPT) was used to assess fine manual dexterity. Participants were instructed to pick the pegs by their affected hand, one by one, and place them in the holes on the pegboard in 30 seconds. The test score 
was the number of pegs placed in the holes. The PPT has a high test-retest reliability [29].

\section{Wolf Motor Function Test (WMFT)}

Wolf Motor Function Test (WMFT) is a standardized performance-based measure of UE motor function which included 15 functional tasks. Each of these tasks is scored according to a 6-point functional ability scale (WMFT FAS) from 0 (not attempted by the affected UE) to 5 (movement seems normal), and a performance time scale (WMFT time). The maximum time given to a subject to complete each functional task was 2 minutes. The mean time of 15 functional tasks was considered for WMFT time. Higher score on WMFT FAS and lower score on WMFT time indicates better UE function. It has been reported that WMFT has a high test-retest and inter-rater reliability in stroke survivors [30].

\section{Statistical analysis}

The normality of data was evaluated by KolmogorovSmirnov test. The stepwise regression models $\left(\mathrm{R}^{2}\right)$ were performed in order to investigate the correlation between predictors and outcomes. In order to determine the relevant factors for each model, firstly, the correlation between predictors and outcomes were evaluated using Pearson and Spearman correlation coefficients for parametric and non-parametric variables, respectively. Only the variables that showed significant correlation with each manual dexterity and UE motor function were included in the respective regression models. The statistical significance was considered at 0.05 .

\section{Results}

In this study, 225 chronic stroke survivors (112 female and 113 male) by mean \pm SD age of $56.46 \pm 12.54$ years

Table 1. Demographics and outcomes in participants

\begin{tabular}{ccc}
\hline Characteristics & Mean \pm SD/Number & 95\% Confidence Interval \\
\hline Sex (female/male) & $112 / 113$ & - \\
\hline Stroke type (ischemia/hemorrhage) & $168 / 57$ & - \\
\hline Affected side (right/left) & $99 / 126$ & - \\
\hline Dominant hand (right/left) & $217 / 8$ & $54.89-57.99$ \\
\hline Age (years) & $56.46 \pm 12.54$ & $3.07-3.78$ \\
\hline Time since stroke (years) & $3.42 \pm 2.66$ & $25.71-26.51$ \\
\hline MMSE (score) & $26.09 \pm 3.05$ & $32.54-37.85$ \\
BBT (number/ 60 s) & $35.13 \pm 21.72$ & $6.02-7.01$ \\
PPT (number/ 30 s) & $6.47 \pm 4.18$ & $40.51-45.40$ \\
WMFT FAS (score) & $42.91 \pm 19.65$ & $40.39-47.03$ \\
WMFT Time (s) & $43.83 \pm 25.89$ & $10.69-11.83$ \\
\hline WPST (degree) & $11.28 \pm 4.49$ & $15.15-17.65$ \\
WEST (score) & $16.40 \pm 9.66$ & $7.63-8.84$ \\
\hline HORT (number of error/ 17) & $8.24 \pm 4.64$ & $7.78-9.15$ \\
\hline HAST (number of correct/ 18) & $8.43 \pm 5.29$ & $10.01-11.63$ \\
s2PD (score) & $10.81 \pm 6.39$ & $9.69-10.42$ \\
\hline m2PD (score) & $9.54 \pm 6.62$ & - \\
\hline
\end{tabular}

Iranian Rehabilitation Journal

MMSE: Mini Mental State Examination; BBT: Box and Block Test; PPT: Purdue Pegboard Test; WMFT: Wolf Motor Function Test; FAS: Functional Ability Scale; WPST: Wrist Position Sense Test; WEST: Weinstein Enhanced Sensory Test; HORT: Haptic Object Recognition Test; HAST: Hand Active Sensation Test; s2PD: static 2-Point Discrimination; m2PD: moving 2-Point Discrimination; SD: Standard Deviation 
Table 2. Correlations between predictors and outcomes (dexterity and upper extremity function) $(\mathrm{n}=225)$

\begin{tabular}{ccccc}
\hline & \multicolumn{3}{c}{ Outcomes } \\
\cline { 2 - 5 } Predictors & $\begin{array}{c}\text { BBT } \\
\text { (Number/ 60 s) }\end{array}$ & $\begin{array}{c}\text { PPT } \\
\text { (Number/ 30 s) }\end{array}$ & $\begin{array}{c}\text { WMFT FAS } \\
\text { (Score) }\end{array}$ & $\begin{array}{c}\text { WMFT } \\
\text { Time (s) }\end{array}$ \\
\hline Sex (female/male) & 0.03 & 0.03 & 0.003 & 0.02 \\
\hline Etiology (ischemia/hemorrhage) & 0.03 & 0.04 & 0.04 & 0.03 \\
\hline Affected side (right/left) & 0.05 & 0.07 & 0.01 & 0.02 \\
\hline Dominant hand (right/left) & 0.003 & 0.003 & 0.02 & 0.02 \\
\hline Age (years) & 0.05 & 0.06 & 0.08 & 0.06 \\
\hline Time since stroke (months) & 0.003 & 0.01 & 0.009 & 0.02 \\
\hline MMSE (score) & 0.03 & 0.03 & 0.03 & 0.05 \\
WPST (degree) & -0.77 & -0.78 & -0.68 & 0.69 \\
\hline WEST (score) & 0.47 & 0.45 & 0.51 & -0.46 \\
\hline HORT (number of error/17) & -0.73 & -0.73 & -0.60 & 0.61 \\
\hline HAST (number of correct/18) & 0.62 & 0.62 & 0.64 & -0.67 \\
S2PD (score) & 0.21 & 0.20 & 0.23 & -0.20 \\
\hline m2PD (score) & 0.56 & 0.56 & 0.64 & -0.59 \\
\hline
\end{tabular}

MMSE: Mini Mental State Examination; BBT: Box-Block Test; PPT: Purdue Pegboard Test; WMFT; Wolf Motor Function Test; FAS, Functional ability scale; WPST, Wrist position sense test; WEST, Weinstein enhanced sensory test; HORT: Haptic Object Recognition Test; HAST: Hand Active Sensation Test; s2PD, static 2-Point Discrimination; m2PD: moving 2-Point Discrimination.

Bold values indicate statistically significant correlation.

and mean $\pm \mathrm{SD}$ time since stroke of $3.42 \pm 2.66$ years participated. Demographic and clinical characteristics of the participants are shown in Table 1. The results showed that sex, stroke type, affected side, dominant hand, age, time since stroke and MMSE score are not significantly correlated with outcomes. However, there was a significant correlation between different somatosensory predictors and motor outcomes (Table 2).

Table 3. A summary of stepwise multiple regression analyses for dexterity

\begin{tabular}{ccccc}
\hline $\begin{array}{c}\text { Functional Balance } \\
\text { Outcomes }\end{array}$ & Predictors/ Models & $\mathbf{R}^{\mathbf{2}(\%)}$ & $\mathbf{R}^{\mathbf{2}}$ Change (\%) & $\mathbf{P}_{(\mathrm{v})}$ \\
\hline & Model 1: WPST & 54.8 & 54.8 & 0.000 \\
BBT & Model 2: WPST+ WEST & 57.8 & 2.9 & 0.000 \\
(number/60 s) & Model 3: WPST+ WEST+ HORT & 59.3 & 1.6 & 0.000 \\
& Model 4: WPST+ WEST+ HORT+ m2PD & 59.9 & 0.8 & 0.000 \\
PPT & Model 1: WPST & 56.6 & 56.6 & 0.000 \\
(number/30 s) & Model 2: WPST+ WEST & 58.6 & 2.00 & 0.000 \\
& Model 3: WPST+ WEST+ HORT & 60.00 & 1.4 & 0.000 \\
\hline
\end{tabular}

Iranian Rehabilitation Journal

BBT: Box-Block Test; PPT: Purdue Pegboard Test; WPST: Wrist position sense test; WEST: Weinstein Enhanced Sensory Test; HORT: Haptic Object Recognition Test; s2PD: static 2-Point Discrimination; m2PD: moving 2-Point Discrimination 
Table 4. A summary of stepwise multiple regression analyses for upper extremity function

\begin{tabular}{|c|c|c|c|c|}
\hline Mobility Outcomes & Predictors/ Models & $\mathbf{R}^{2}(\%)$ & $\mathrm{R}^{2}$ change (\%) & $\mathbf{P}$ \\
\hline \multirow{5}{*}{ WMFT FAS (score) } & Model 1: WPST & 38.8 & 38.8 & 0.000 \\
\hline & Model 2: WPST+ WEST & 45.00 & 6.3 & 0.000 \\
\hline & Model 3: WPST+ WEST+ m2PD & 49.4 & 4.4 & 0.000 \\
\hline & Model 4: WPST+ WEST+ m2PD+ HAST & 50.6 & 1.2 & 0.000 \\
\hline & Model 5: WPST+ WEST+ m2PD+ HAST+ HORT & 51.8 & 1.1 & 0.000 \\
\hline \multirow{5}{*}{ WMFT (time/s) } & Model 1: WPST & 39.8 & 39.8 & 0.000 \\
\hline & Model 2: WPST+ WEST & 43.3 & 3.5 & 0.000 \\
\hline & Model 3: WPST+ WEST+ m2PD & 46.00 & 2.6 & 0.000 \\
\hline & Model 4: WPST+ WEST+ m2PD+ HAST & 48.2 & 2.3 & 0.000 \\
\hline & Model 5: WPST+ WEST+ m2PD+ HAST+ HORT & 49.5 & 1.3 & 0.000 \\
\hline
\end{tabular}

Iranian Rehabilitation Journal

WMFT: Wolf Motor Function Test; FAS: Functional Ability Scale; WPST: Wrist Position Sense Test; WEST: Weinstein Enhanced Sensory Test; s2PD: static 2-Point Discrimination; m2PD: moving 2-Point Discrimination; HAST: Hand Active Sensation Test; HORT: Haptic Object Recognition Test

The regression models explained up to $59.9 \%$ of the variance for gross manual dexterity (i.e. BBT) and $60.7 \%$ for the fine manual dexterity (i.e. PPT). In all stepwise models for manual dexterity, the wrist Proprioception, measured by WPST, was the strongest predictor. The light touch threshold (i.e. WEST) explained the second highest level of the variance for both fine and gross manual dexterity. The HORT and m2PD explained a small percentage of the variance in both fine and gross manual dexterity (Table 3 ).

Further, the regression models explained up to $51.8 \%$ and $49.5 \%$ of the variance in functional ability of the affected UE (i.e. WMFT FAS) and the time to perform functional task (i.e. WMFT time), respectively. The wrist Proprioception was the strongest predictor for UE motor function in all stepwise models, followed by WEST, m2PD, HAST and HORT (Table 4).

\section{Discussion}

Despite high prevalence of somatosensory deficits in stroke survivors and the important role of somatosensory inputs in motor learning and control, the researches to date have tended to focus on motor impairments of stroke survivors and their contributing role in UE motor function than somatosensory deficits. By comprehensively investigating the effects of different types of Somatosensation (in both lower and higher-order levels) on manual dexterity and UE motor function in chronic stroke survivors, we found that wrist Proprioception was the strongest contributing factor followed by light touch threshold. To the best of the authors' knowledge, this is the first study surveying the underlying association between lower and higher-order Somatosensations and manual dexterity as well as UE motor function in such a large sample of chronic stroke survivors.

The results of this study showed that wrist Proprioception (as measured by WPST) was the strongest predictor for both gross and fine manual dexterity as well as UE motor function in chronic stroke survivors. This result indicates the need to consider wrist Proprioception in rehabilitation for improving manual dexterity and UE motor function in these patients. The WPST correlated negatively with BBT, PPT, and WMFT FAS while it correlated positively with WMFT time. It means that chronic stroke survivors, who had greater impairment of wrist Proprioception (i.e. higher score on WPST), had less gross and fine manual dexterity and less ability to perform UE functional task, and they needed further time to complete these functional task. Performing BBT, PPT, and most of the functional task of WMFT involves wrist movements. Moreover, it has been shown that proprioceptive information (especially limb position sense, which is measured by WPST in the current study) play an important role in different aspects of motor function including sequencing multi-joint movements [10, 11], 
control of goal-directed movements [10] and correcting ongoing movements [12]. Thus, the significant contributing effect of wrist Proprioception on manual dexterity and UE motor function is not surprising. This result is consistent with the results of Scalha et al. (2011) and Meyer et al. (2015) who found a significant correlation between different UE joints Proprioception and motor subscale of FMA of UE [14, 16]. Park et al. (2008) also reported a significant association between Proprioception (as measured by sensory subscale of FMA) and UE function measured by motor activity $\log$ (MAL) in stroke survivors [17].

The light touch threshold (as measured by WEST) was the second most significant predictor which was included in the final regression models for both manual dexterity and UE motor function. This finding suggests that in addition to wrist Proprioception, light touch should be considered as a therapeutic target for improving manual dexterity and UE motor function in chronic stroke survivors. One possible explanation for this result might be that reaching and grasping movements, required for performing BBT, PPT, and some of the functional task in WMFT, needs light touch inputs for coordinating and modulating the output force balance [31]. Furthermore, Bourane et al. (2015) recently found a sensorimotor circuits in the spinal cord and cerebellum through which the light touch inputs act as a feedback for fine dynamic motor control [32]. Bowden et al. (2014) also reported significant correlation between light touch threshold measured by von Fery monofilaments and WMFT time $(\mathrm{r}=0.31-0.37, \mathrm{P}<0.05)$ in stroke survivors [33].

In addition, Meyer et al. (2016) found that perceptual threshold of touch was significantly correlated with UE motor impairments ( $r=-0.60$ to -0.66$)$ in stroke survivors [15]. However, Shamay et al. (2011) did not find significant association between light touch threshold measured by Semmes-Weinstein monofilaments and affected upper extremity function measured by Jebson-Taylor test in stroke survivors [13]. One possible explanation for this discrepancy may be that Shamay et al. tested light touch in three sites (tip of index finger, $1 \mathrm{~cm}$ below the metacarpophalangeal joint of middle finger on the dorsal side and center of palm) that may not be representative of whole tactile deficit of stroke survivors. Moreover, different measure of UE motor function and small sample size $(n=19)$ may also impact the Shamay et al. results.

The haptic performance (as measured by HORT), tactile discrimination (as evaluated by 2PD) and the ability to discriminate weight and texture (as assessed by HAST) showed a significant correlation with manual dexterity and UE motor function in the current study. However, the HORT and m2PD accounted for a small part of the variance in the final regression models for both manual dexterity and UE motor function. Moreover, the HAST was only included in the final regression models for UE motor function (i.e. WMFT FAS and time). The clinical implication of these findings is that if a chronic stroke survivor has difficulties in identifying spatial characteristics of a tactile stimulus or its location, these difficulties could affect her/his manual dexterity and ability to perform functional tasks as well as the time needed to perform these tasks.

The effect of haptic performance and tactile, weight and texture discrimination on manual dexterity and UE motor function may be explained by the fact that impairments of the Somatosensation can affect the ability to identify different characteristics of an object through touch, leading to impaired spontaneous hand use, object manipulation, ability to calibrate grip force for hand movements and relearning motor skills [9, 34]. Williams et al. (2006) showed that HAST score was significantly correlated with WPST score [26], hence, the effect of wrist Proprioception may dominate the effect of weight and texture discrimination on manual dexterity and UE motor function. Meyer et al. (2015) also reported low correlation between 2PD and motor subscale of FMA [14]. Further, Smith et al. (2009) showed that improvements of tactile discrimination (as measured by 2PD) and haptic object recognition were associated with improvement of UE motor function of chronic stroke survivors evaluated by WMFT [35]. However, Scalha et al. (2011) found that weight discrimination and tactile recognition of objects did not significantly correlated with motor score of FMA in chronic stroke survivors [16]. This inconsistency may be due to different chosen somatosensory and motor function measures and small sample size of Scalha et al. study.

Another finding of this study was that manual dexterity and UE motor function of chronic stroke survivors did not significantly correlated with sex, stroke type, affected side, dominant hand, age, and time since stroke. This finding is consistent with that of Shamay et al. (2011) who found that age and time since stroke did not significantly correlated with the motor function of affected hand [13].

\section{Conclusion}

The results of this study showed that wrist Proprioception was the strongest predictor for manual dexterity and UE motor function in chronic stroke survivors, followed by light touch as well as haptic performance and tactile, 
weight and texture discrimination, but to a lesser extent. Treatment of somatosensory deficits has not been a significant focus for chronic stroke survivors. However, because of the main contribution of somatosensation in the variance of manual dexterity and UE motor function of stroke survivors, it would seem necessary that targeting somatosensory impairments, especially wrist Proprioception and light touch, becomes an important part of rehabilitation for chronic stroke survivors.

\section{Ethical Considerations}

\section{Compliance with ethical guidelines}

The study was approved by the Ethics Committee of Iran University of Medical Sciences (IR.IUMS.res. 1394. 9311355010). All patients agreed to participate and signed a consent form.

\section{Funding}

This study was financially supported by Iran University of Medical Sciences (IUMS); Tehran, Iran. This article is part of MSc. thesis of first author in the Department of Occupational Therapy, School of Rehabilitation Sciences, Iran University of Medical Sciences.

\section{Conflict of interest}

The authors declare no conflict of interest.

\section{Acknowledgements}

The authors would like to thank all people who participated in this study.

\section{References}

[1] Nakayama H, Jørgensen HS, Raaschou HO, Olsen TS. Recovery of upper extremity function in stroke patients: The Copenhagen stroke study. Archives of Physical Medicine and Rehabilitation. 1994; 75(4):394-8. [DOI:10.1016/00039993(94)90161-9]

[2] Sveen U, Bautz-Holter E, Sodring KA, Wyller TO, Laake K. Association between impairments, self-care ability and social activities 1 year after stroke. Disability and Rehabilitation. 1999; 21(8):372-7. [DOI:10.1080/096382899297477] [PMID]

[3] Meyer S, Karttunen AH, Thijs V, Feys H, Verheyden G. How do somatosensory deficits in the arm and hand relate to upper limb impairment, activity, and participation problems after stroke: A systematic review. Physical Therapy. 2014; 94(9):1220-31. [DOI:10.2522/ptj.20130271] [PMID]

[4] Squire L, Berg D, Bloom FE, du Lac S, Ghosh A, Spitzer NC. Fundamental neuroscience: The somatosensory system. Waltham, MA: Academic Press; 2008.

[5] Carey LM. Stroke rehabilitation: Insights from neuroscience and imaging. Oxford: Oxford University Press; 2012. [DOI:10.1093/med/9780199797882.001.0001]

[6] Doyle S, Fasoli SE, McKenna KT. Interventions for sensory impairment in the upper limb after stroke. In: Doyle S (ed), Cochrane database of systematic reviews. Hoboken: John Wiley \& Sons, Ltd; 2007. [DOI:10.1002/14651858.CD006331]

[7] Carey LM. Somatosensory loss after stroke. Critical Reviews ${ }^{\mathrm{TM}}$ in Physical and Rehabilitation Medicine. 1995; 7(1):51-91. [DOI: 10.1615/critrevphysrehabilmed.v7.i1.40]

[8] Shah SK. Deficits affecting the function of the paralyzed arm following hemiplegia. Australian Occupational Therapy Journal. 1978; 25(2):12-9. [DOI:10.1111/j.1440-1630.1978. tb00656.x]

[9] Carey LM, Matyas TA, Oke LE. Sensory loss in stroke patients: Effective training of tactile and proprioceptive discrimination. Archives of Physical Medicine and Rehabilitation. 1993; 74(6):602-11. [DOI:10.1016/0003-9993(93)90158-7]

[10] Rothwell JC, Traub MM, Day BL, Obeso JA, Thomas PK, Marsden CD. Manual motor performance in a deafferented man. Brain. 1982; 105(3):515-42. [DOI:10.1093/ brain/105.3.515] [PMID]

[11] Cordo PJ. Kinesthetic control of a multipoint movement sequence. Journal of Neurophysiology. 1990; 63(1):161-72 [DOI:10.1152/jn.1990.63.1.161] [PMID]

[12] Hasan Z. Role of proprioceptors in neural control. Current Opinionin Neurobiology.1992;2(6):824-9. [DOI:10.1016/09594388(92)90140-G]

[13] Shamay NS, William TW, Patrick KW, Philip TT, Jefferry WC. Sensorimotor impairments of paretic upper limb correlates with activities of daily living in subjects with chronic stroke. South African Journal of Physiotherapy. 2011; 67(1):916. [DOI:10.4102/sajp.v67i1.34]

[14] Meyer S, De Bruyn N, Lafosse C, Van Dijk M, Michielsen $\mathrm{M}$, Thijs L. Somatosensory impairments in the upper limb post-stroke: Distribution and association with motor function and visuospatial neglect. Neurorehabilitation and Neural Repair. 2016; 30(8):731-42. [DOI:10.1177/1545968315624779] [PMID]

[15] Meyer S, De Bruyn N, Krumlinde-Sundholm L, Peeters A, Feys H, Thijs V. Associations between sensorimotor impairments in the upper limb at 1 week and 6 months after stroke. Journal of Neurologic Physical Therapy. 2016; 40(3):186-95. [DOI:10.1097/NPT.0000000000000138] [PMID]

[16] Scalha TB, Miyasaki E, Lima NM, Borges G. Correlations between motor and sensory functions in upper limb chronic hemiparetics after stroke. Arquivos de Neuro-Psiquiatria 2011; 69(4):624-9. [DOI:10.1590/S0004-282X2011000500010] [PMID]

[17] Park SW, Wolf SL, Blanton S, Winstein C, Nichols Larsen DS. The excite trial: Predicting a clinically meaningful motor activity log outcome. Neurorehabilitation and Neural Repair 
2008; 22(5):486-93. [DOI:10.1177/1545968308316906] [PMID] [PMCID]

[18] Azad A, Taghizadeh G, Mohammadian E, Mohmmadinezhad T, Lajevardi L. Persian translation and test-retest reliability of the Activities-specific Balance Confidence Scale in Iranian Chronic Stroke. Journal of Modern Rehabilitation. 2017; 10(2):74-9.

[19] Azad A, Mehraban AH, Mehrpour M, Mohammadi B. Clinical assessment of fear of falling after stroke: validity, reliability and responsiveness of the Persian version of the Fall Efficacy Scale-International. Medical Journal of the Islamic Republic of Iran. 2014; 28:131. [PMCID]

[20] Bailey MJ, Riddoch MJ, Crome P. Evaluation of a test battery for hemineglect in elderly stroke patients for use by therapists in clinical practice. Neurorehabilitation. 2000; 14(3):13950. [PMID]

[21] Daneshjoo F, Azad A, Mandehgary M, Mehdizadeh M, Taghizadeh G. Correlation between lower and higher order sensory functions and manual dexterity in dominant and non-dominant hand of patients with idiopathic Parkinson's disease. Journal of Basic and Clinical Pathophysiology. 2016; 4(2):27-36.

[22] Taghizadeh G, Azad A, Kashefi S, Fallah S, Daneshjoo F. The effect of sensory-motor training on hand and upper extremity sensory and motor function in patients with idiopathic Parkinson disease. Journal of Hand Therapy. 2017; 1-7. [DOI:10.1016/j.jht.2017.08.001]

[23] Arakawa T, Otao H, Okamura M, Ono T. Reliability of the Semmes-Weinstein monofilaments test in stroke patients. Japanese Journal of Health Promotion and Physical Therapy. 2012; 2(2):65-68. [DOI:10.9759/hppt.2.65]

[24] Carey LM, Oke LE, Matyas TA. Impaired limb position sense after stroke: A quantitative test for clinical use. Archives of Physical Medicine and Rehabilitation. 1996; 77(12):1271-8. [DOI:10.1016/S0003-9993(96)90192-6]

[25] Wolny T, Linek P, Michalski P. Inter-rater reliability of twopoint discrimination in acute stroke patients. Neurorehabilitation. 2017; 1-8. [DOI:10.3233/NRE-171464]

[26] Williams PS, Basso DM, Case Smith J, Nichols Larsen DS, Development of the Hand Active Sensation Test: Reliability and validity. Archives of Physical Medicine and Rehabilitation. 2006; 87(11):1471-7. [DOI:10.1016/j.apmr.2006.08.019] [PMID]

[27] Norman JF, Crabtree CE, Norman HF, Moncrief BK, Herrmann M, Kapley N. Aging and the visual, haptic, and crossmodal perception of natural object shape. Perception. 2006; 35(10):1383-95. [DOI:10.1068/p5504] [PMID]

[28] Desrosiers J, Bravo G, Hébert R, Dutil É, Mercier L. Validation of the Box and Block Test as a measure of dexterity of elderly people: Reliability, validity, and norms studies. Archives of Physical Medicine and Rehabilitation. 1994 75(7):751-5. [PMID]

[29] Azad A, Taghizadeh G, Ghorbanpoor H, Lajevardi L, Farhadian M. Relationship between laterality and handedness with the higher order sensory functions and manual dexterity of the elderly. Iranian Rehabilitation Journal, 2017; 15(4):367-76. [DOI:10.29252/nrip.irj.15.4.367]
[30] Wolf SL, Catlin PA, Ellis M, Archer AL, Morgan B, Piacentino A. Assessing Wolf Motor Function Test as outcome measure for research in patients after stroke. Stroke. 2001; 32(7):1635-9. [DOI:10.1161/01.STR.32.7.1635] [PMID]

[31] Roby Brami A, Fuchs S, Mokhtari M, Bussel B. Reaching and grasping strategies in hemiparetic patients. Motor Control. 1997; 1(1):72-91. [DOI:10.1123/mcj.1.1.72]

[32] Bourane S, Grossmann KS, Britz O, Dalet A, Del Barrio MG, Stam FJ. Identification of a spinal circuit for light touch and fine motor control. Cell. 2015; 160(3):503-15. [DOI:10.1016/j. cell.2015.01.011] [PMID] [PMCID]

[33] Bowden JL, Lin GG, McNulty PA. The prevalence and magnitude of impaired cutaneous sensation across the hand in the chronic period post-stroke. PLOS ONE. 2014; 9(8):e104153. [DOI:10.1371/journal.pone.0104153] [PMID] [PMCID]

[34] Gordon AM, Charles J, Steenbergen B. Fingertip force planning during grasp is disrupted by impaired sensorimotor integration in children with hemiplegic cerebral palsy. Pediatric Research. 2006; 60(5):587-91. [DOI:10.1203/01. pdr.0000242370.41469.74] [PMID]

[35] Smith PS, Dinse HR, Kalisch T, Johnson M, Walker Batson D. Effects of repetitive electrical stimulation to treat sensory loss in persons post-stroke. Archives of Physical Medicine and Rehabilitation. 2009; 90(12):2108-11. [DOI:10.1016/j. apmr.2009.07.017] [PMID] 
